\title{
Relativistic Dynamics of a Quantum System
}

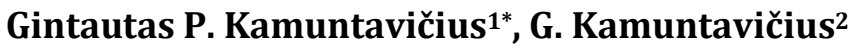 \\ ${ }^{1}$ Department of Physics, Vytautas Magnus University, Kaunas, Lithuania \\ ${ }^{2}$ Christ's college, University of Cambridge, Cambridge, UK \\ Email: ^gintautas.kamuntavicius@vdu.lt
}

How to cite this paper: Kamuntavičius, G.P. and Kamuntavičius, G. (2017) Relativistic Dynamics of a Quantum System. Journal of Applied Mathematics and Physics, 5, 1478-1492.

https://doi.org/10.4236/jamp.2017.57121

Received: June 19, 2017

Accepted: July 21, 2017

Published: July 24, 2017

Copyright (C) 2017 by authors and Scientific Research Publishing Inc. This work is licensed under the Creative Commons Attribution International License (CC BY 4.0).

http://creativecommons.org/licenses/by/4.0/

\begin{abstract}
In this paper, we examine quantum systems with relativistic dynamics. We show that for a successful description of these systems, the application of Galilei invariant nonrelativistic Hamiltonian is necessary. To modify this Hamiltonian to relativistic dynamics, we require precise relativistic kinetic energy operators instead of nonrelativistic ones for every internal (Jacobi) coordinate. Finally, we introduce and investigate the Schrödinger equation with relativistic dynamics for two-particle systems with harmonic oscillator and Coulomb potentials.
\end{abstract}

\section{Keywords}

Special Relativity, Quantum Mechanics, Relativistic Wave Equations, Solutions of Wave Equations: Bound States

\section{Introduction}

The definition of relativistic quantum mechanical momentum operator, introduced in [1], allows us to modify the Schrödinger equation for free particle, taking into account basic ideas of special relativity theory (SRT). However, the most important and interesting problem is the application of relativistic dynamics to a many-particle system. To solve it, we have to carefully investigate the main conclusions of SRT and quantum mechanics to find the possibility of consistence of both theories.

The core of SRT are two postulates [2]. The first is Galilei invariance, stating equivalence of different inertial reference systems, and the second is the statement that speed of light $c$ is constant in all systems of this kind. The main results of the theory are:

- the mass of particle $m$ (sometimes called as rest mass) is constant in all reference frames;

- the relativistic momentum of particle, moving in reference frame with 
velocity $v$, is $p=\gamma m v$, where $\gamma=\left(1-v^{2} / c^{2}\right)^{-1 / 2}$ is the Lorentz factor;

- the total energy of the particle equals $E=\gamma m c^{2}$, and the kinetic energy is $T=(\gamma-1) m c^{2}$;

- the general relation between radius vector $\boldsymbol{r}$ and time $t$ of the event as seen in the reference frame $S$ and the radius vector $\boldsymbol{r}^{\prime}$ and time $t^{\prime}$ of the same event in the reference frame $S^{\prime}$, which is moving with uniform velocity relative to $S$, is defined by Lorentz transformation, which implies invariance of the space-time interval, defined as the scalar product of these four-vectors:

$$
s^{2}=(c t)^{2}-\boldsymbol{r}^{2}=\left(c t^{\prime}\right)^{2}-\left(\boldsymbol{r}^{\prime}\right)^{2} ;
$$

- the mass invariance with respect to transformations from one to another inertial reference frame of the above type also defines invariance of the fourvector, composed of relativistic energy and relativistic momentum:

$$
m^{2} c^{4}=E^{2}-(p c)^{2}=\left(E^{\prime}\right)^{2}-\left(p^{\prime} c\right)^{2} .
$$

The main problem is-how and to what extent can these statements be applied in the theory of quantum systems?

\section{Quantum System's Schrödinger Equation}

The base for modifications is the many-particle Schrödinger equation

$$
i \hbar \frac{\partial}{\partial t} \Psi\left(\rho_{1}, \rho_{2}, \cdots, \rho_{N}, t\right)=\hat{H}\left(\rho_{1}, \rho_{2}, \cdots, \rho_{N}\right) \Psi\left(\rho_{1}, \rho_{2}, \cdots, \rho_{N}, t\right),
$$

written in a laboratory reference system. Here $\rho_{j}$ marks the set of one-particle variables (spatial radius vector $\boldsymbol{r}_{j}$ and internal degrees of freedom $\tau_{j}$ like mass, charge, spin, isospin and so on for every particle). The Hamiltonian is independent of time, so the formal solution of (3) is

$$
\Psi\left(\rho_{1}, \rho_{2}, \cdots, \rho_{N}, t\right)=\exp \left(-\frac{i}{\hbar} \hat{H} t\right) \Phi\left(\rho_{1}, \rho_{2}, \cdots, \rho_{N}\right) .
$$

The stationary states of (3) correspond to the functions $\Phi$, defined as the eigenfunctions of the Hamiltonian:

$$
\hat{H} \Phi=E \Phi .
$$

The probability density is therefore independent of time. The quantum system is stable with respect to breakup into composing particles if the corresponding eigenvalue $E$ is negative ( $-E$ equals the binding energy of the system). We will consider here the application of relativistic dynamics to quantum systems defined this way.

The introduced presentation gives the simplest way to ensure symmetry of the Hamiltonian with respect to permutations of one-particle degrees of freedom, because quantum systems or their subsystems are composed of identical particles. Moreover, these particles are fermions, so the eigenfunction of the Hamiltonian has to be antisymmetric with respect to permutations of the variables of identical fermions. It is especially simple to project the wave function, written in single-particle variables, to the corresponding subspace of 
Hilbert space. Various methods for antisymmetrization of wave functions, dependent on single-particle coordinates, are thoroughly developed from application of Slater determinants or Racah coefficients of fractional parentage [3] [4], to algorithms, devoted for operations with wave functions of this kind, such as second quantization and formalism applying particles creation and annihilation operators.

The relativistic dynamics introduction for Schrödinger Equation (3), if even possible, is an immensely difficult problem. The equation is written in a laboratory reference system. Due to the necessary equivalence of all inertial reference systems, the equation has to be invariant with respect to time and space variables for the Lorentz transformation of every particle, keeping invariant the length of the four vector, defined by Equation (1). Even for one free particle such an equation (known as the Dirac equation) is possible only after defining the quantum mechanical momentum operator as corresponding to the relativistic momentum. However, this operator does not have any dependence on velocity, necessary for relativistic momentum, so the corresponding equation can not be considered completely relativistic.

Moreover, even in the nonrelativistic approximation the wave functions defined in this way are not acceptable for a precise description of the quantum system. These functions do not satisfy Galilei invariance and, in many cases, contains uncontrolled center of mass excitations. For the best illustration of this statement, let us consider a two-particle system. The potential energy operator is translationally invariant, i.e. independent of the center of mass coordinate, hence the only problem is in the kinetic energy operator. The corresponding wave function written in one-particle spatial variables is not under application, because the elimination of the center of mass kinetic energy operator in a two-particle Hamiltonian is an easy task. Elimination of the center of mass kinetic energy operator is not complicated for a system of $N$ particles either. However, after this the Hamiltonian of the quantum system appears as a function of $3(N-1)$ internal spatial variables and $N$ sets of individual degrees of freedom of particles and antisymmetrization of eigenfunctions of this operator becomes a very complex problem.

Therefore, the solution of the nonrelativistic Schrödinger equation for a quantum system with internal Hamiltonian is a real challenge, but only these wave functions can give an adequate description of the quantum system. The experimental investigation of a quantum system is based on observing the behavior of a system after excitation by an external agent. Every act of this kind excites the center of mass of the system and, at the same time, the internal degrees of freedom. Unfortunately, control of the center of mass movement after a collision is very problematic. Among the attempts to minimize the energy loss due to center of mass excitation most notable are the Mösbauer effect and colliding beams accelerators. To avoid gamma ray energy loss due to nuclear recoil, Mösbauer used nuclei bound in a solid. On the other hand, in an experiment involving head-on colliding beams with identical particles and equal 
energies, the kinetic energy of the center of mass is zero before, and hence after, the collision. Either way, as the exciting particle leaves the range of the interaction force, the center of mass velocity is constant, so this point can serve as the origin of an inertial reference frame. All particles, composing the system, move in the same inertial frame, hence any transformations of radius vector and time coordinate or energy and momentum of given particle between different inertial systems are not necessary. Both the nonrelativistic and modified relativistic (introduced in [1]) internal Hamiltonians are invariant with respect to translations. The energy of a stationary state is defined precisely, hence the energy and momentum conservation law (2) is senseless in a fixed reference frame defined for a quantum system by its internal Hamiltonian. Moreover, at a precisely defined energy the time coordinate is completely uncertain, therefore it is not necessary as argument of wave function. At the same time in formalisms, operating with one particle coordinates, all inertial systems have to be equivalent and invariance of formalism with respect to Lorentz transformations is necessary. The space-time interval, defined by scalar product of four-dimensional vectors and invariant with respect to transformations between different inertial frames, requires every particle to have different spatial and time variables. The problems of wave functions presented this way are well known (lattice QCD; for a review, see [5]). In our formalism we don't have to worry about interval and mass invariance in different inertial reference systems. Instead, we just modify the internal Hamiltonian of a quantum system by introducing the relativistic kinetic energy operator instead of the nonrelativistic one for every internal spatial coordinate.

The transformation of the initial Schrödinger Equation (3), present in a laboratory system, to the aforementioned form, starts with the nonrelativistic Hamiltonian transformation to center of mass and internal variables. The canonical choice is the Jacobian coordinates, defined as in [6]. The nonrelativistic kinetic energy operator, expressed in these coordinates, retains the usual form:

$$
\sum_{j=1}^{N} \frac{1}{m_{j}} \Delta_{j}=\sum_{\beta=0}^{N-1} \frac{1}{\mu_{\beta}} \Delta_{(\beta)} .
$$

Here $\beta$ is the subscript of the Jacobi variable $\xi_{\beta}$, the corresponding Laplasian is $\Delta_{(\beta)}$, and reduced mass equals $\mu_{\beta}$. The Jacobi coordinate $\xi_{0}$ and mass $\mu_{0}$ are defined as the system's center of mass radius vector and mass:

$$
\xi_{0}=\frac{1}{\mu_{0}} \sum_{j=1}^{N} m_{j} \boldsymbol{r}_{j}, \quad \mu_{0}=\sum_{j=1}^{N} m_{j} .
$$

The Hamiltonian appears with separated center of mass and internal variables:

$$
\hat{H}\left(\rho_{1}, \rho_{2}, \cdots, \rho_{N}\right)=\hat{H}_{c m}\left(\xi_{0}\right)+\hat{H}_{i n t}\left(\xi_{1}, \xi_{2}, \cdots, \xi_{N-1}, \tau_{1}, \tau_{2}, \ldots, \tau_{N}\right) .
$$

Furthermore, to transform to a relativistic form, we need to take into account the rest energies of the particles in the Hamiltonian:

$$
\sum_{j=1}^{N} m_{j} c^{2}=\mu_{0} c^{2}
$$


Now the quantum system's Schrödinger Equation (3) takes the following form:

$$
\left(i \hbar \frac{\partial}{\partial t}-\hat{H}_{c m}-\mu_{0} c^{2}-\hat{H}_{\mathrm{int}}\right) \Psi\left(\xi_{0}, \xi_{1}, \cdots, \xi_{N-1}, \tau_{1}, \tau_{2}, \cdots, \tau_{N}, t\right)=0
$$

The variables of the complete Hamiltonian are separated, so the simplest solutions of this equation are products of functions, dependent on different variables:

$$
\Psi\left(\xi_{0}, \xi_{1}, \cdots, \xi_{N-1}, \tau_{1}, \tau_{2}, \cdots, \tau_{N}, t\right)=\Psi_{c m}\left(\xi_{0}, t\right) \Psi_{\text {int }}\left(\xi_{1}, \xi_{2}, \cdots, \xi_{N-1}, \tau_{1}, \tau_{2}, \cdots, \tau_{N}\right),
$$

where the introduced functions are eigenfunctions of the following operators, corresponding to the same eigenvalue $E$ :

$$
\left(i \hbar \frac{\partial}{\partial t}-\hat{H}_{c m}-\mu_{0} c^{2}-E\right) \Psi_{c m}\left(\xi_{0}, t\right)=0
$$

and

$$
\left[\hat{H}_{i n t}-E\right] \Psi_{i n t}\left(\xi_{1}, \xi_{2}, \cdots, \xi_{N-1}, \tau_{1}, \tau_{2}, \cdots, \tau_{N}\right)=0
$$

The eigenvalue $E$, by definition of the internal Hamiltonian, equals the binding energy of system with opposite sign. Thus $\mu_{0} c^{2}+E$ is the reduced, slightly smaller, rest energy of the bound system $\bar{\mu}_{0} c^{2}$. Necessarily, the kinetic energy operator has to be expressed with the reduced rest energy of system, i.e. as

$$
\hat{H}_{c m}=-\frac{(\hbar c)^{2}}{\bar{\mu}_{0} c^{2}} \Delta_{(0)} .
$$

We go on to further modify the center of mass Schrödinger Equation (12), taking into account the relativistic dynamics, by applying the technique introduced in [1].

It is necessary to modify the kinetic energy operators for all Jacobi variables for the introduction of relativistic dynamics into the internal Hamiltonian. The natural subject for the application of these ideas is the two-particle system.

\section{Internal Hamiltonian of a Two-Particle System}

The internal Hamiltonian of a two-particle system, whose interaction is defined by a local potential, dependent on the radius vector $\boldsymbol{r}=\boldsymbol{r}_{2}-\boldsymbol{r}_{1}$ (here $\boldsymbol{r}_{2}$ and $\boldsymbol{r}_{1}$ are radius vectors, defining locations of particles in laboratory system) and on internal degrees of freedom of particles $\tau_{1}$ and $\tau_{2}$, is

$$
\hat{H}_{0}\left(\boldsymbol{r}, \tau_{1}, \tau_{2}\right)=\hat{T}^{(0)}(\boldsymbol{r}, \mu)+\hat{V}\left(\boldsymbol{r}, \tau_{1}, \tau_{2}\right) .
$$

Here $\hat{T}^{(0)}$ is the nonrelativistic kinetic energy operator

$$
\hat{T}^{(0)}(\boldsymbol{r}, \mu)=-\frac{\hbar^{2}}{2 \mu} \Delta=-\frac{(\hbar c)^{2}}{2 \mu c^{2}}\left[\frac{1}{r} \frac{\partial^{2}}{\partial r^{2}} r-\frac{\hat{L}^{2}(\theta \varphi)}{r^{2}}\right],
$$

with the reduced mass of system $\mu=m_{1} m_{2} /\left(m_{1}+m_{2}\right)$ and the orbital momentum operator $\hat{L}^{2}(\theta \varphi)$. The eigenvalues of the stationary Schrödinger 
equation with this Hamiltonian due to symmetries of potential are identified at least by such quantum numbers as parity $\pi$ and total angular momentum $J$. The simpler is the potential, the larger is the set of exact quantum numbers, characterizing the eigenfunction of this Hamiltonian, i.e. the state of quantum system.

The Hamiltonian with relativistic dynamics

$$
\hat{H}\left(\boldsymbol{r}, \tau_{1}, \tau_{2}\right)=\hat{T}(\boldsymbol{r}, \mu)+\hat{V}\left(\boldsymbol{r}, \tau_{1}, \tau_{2}\right),
$$

is defined with the relativistic kinetic energy operator

$$
\hat{T}(\boldsymbol{r}, \mu)=\mu c^{2}\left[\left(1-\frac{2 \hat{T}^{(0)}(\boldsymbol{r}, \mu)}{\mu c^{2}}\right)^{-1 / 2}-1\right] .
$$

This Hamiltonian is translationally invariant, describing the relative movement of particles in an inertial reference frame, whose origin is at the center of mass of system. The eigenfunctions of the Hamiltonian (17), as in the nonrelativistic case, can be identified by the same set of exact quantum numbers, because the operators $\hat{H}_{0}\left(\boldsymbol{r} \tau_{1} \tau_{2}\right)$ and $\hat{H}\left(\boldsymbol{r}, \tau_{1}, \tau_{2}\right)$ share the common set of commuting operators.

However, a new problem appears. In general, the eigenfunctions of the nonrelativistic Hamiltonian and the Hamiltonian with relativistic dynamics are different due to presence of potential. For a free particle they coincide, but here the possibility to define a relation of eigenvalues of these Hamiltonians is not as simple. Let us restrict the investigation by applying wave functions, obtained solving the nonrelativistic Schrödinger equation. The nonrelativistic energy expectation value equals

$$
E^{(0)}=\left\langle\hat{T}^{(0)}(\boldsymbol{r}, \mu)\right\rangle+\left\langle\hat{V}\left(\boldsymbol{r}, \tau_{1}, \tau_{2}\right)\right\rangle
$$

The corrected value for this wave function is

$$
E=\langle\hat{T}(\boldsymbol{r}, \mu)\rangle+\left\langle\hat{V}\left(\boldsymbol{r}, \tau_{1}, \tau_{2}\right)\right\rangle .
$$

For a relativistic kinetic energy expectation value evaluation we will apply three different approximations.

The first approximation is based on a series expansion of the relativistic kinetic energy operator:

$$
\hat{T}(\boldsymbol{r}, \mu)=\hat{T}^{(0)}(\boldsymbol{r}, \mu)+\frac{3\left(\hat{T}^{(0)}(\boldsymbol{r}, \mu)\right)^{2}}{2 \mu c^{2}}+\cdots,
$$

where we can successfully evaluate the expectation value of $\left(\hat{T}^{(0)}(\boldsymbol{r}, \mu)\right)^{2}$ and therefore the kinetic energy with first order correction

$$
T^{(1)}=\left\langle\hat{T}^{(0)}\right\rangle+\frac{3}{2 \mu c^{2}}\left\langle\left(\hat{T}^{(0)}\right)^{2}\right\rangle
$$

can be successfully evaluated. This result is precise enough for quantum systems with dynamics not far from nonrelativistic.

The second approximation is 


$$
T^{(2)}=\mu c^{2}\left[\left(1-\frac{2\left\langle\hat{T}^{(0)}\right\rangle}{\mu c^{2}}\right)^{-1 / 2}-1\right] .
$$

With this approximation, we ensure, according to the main idea of SRT, the expectation value of the nonrelativistic kinetic energy to be less than $\mu c^{2} / 2$, same as for a free particle. It is the really original result, consistent with the basic postulate of SRT stating that the relative velocity of particles cannot be larger than speed of light at any interaction. However, this result is only exact in the case when the eigenfunctions of the kinetic energy operator are applied for the calculation of the expectation value. For a quantum system this approximation at a low nonrelativistic kinetic energy gives a lower bound for relativistic kinetic energy expectation value due to a relation, valid in an arbitrary basis:

$$
\left\langle\left(\hat{T}^{(0)}\right)^{2}\right\rangle \geq\left\langle\hat{T}^{(0)}\right\rangle^{2} \text {. }
$$

We obtain the third evaluation of relativistic kinetic energy in a given state of a quantum system by applying spectral representation of the corresponding operator. Both kinetic energy operators have the same set of eigenfunctions. The internal Schrödinger equation for the nonrelativistic operator is

$$
\hat{T}^{(0)}(\boldsymbol{r}) \psi_{\boldsymbol{k}}(\boldsymbol{r})=T_{k}^{(0)} \psi_{\boldsymbol{k}}(\boldsymbol{r}) .
$$

We can rewrite it as

$$
\Delta \psi_{k}(\boldsymbol{r})=-k^{2} \psi_{\boldsymbol{k}}(\boldsymbol{r}),
$$

where

$$
k^{2}=\frac{2 \mu c^{2} T_{k}^{(0)}}{(\hbar c)^{2}}
$$

and

$$
\psi_{\boldsymbol{k}}(\boldsymbol{r})=\frac{1}{(2 \pi)^{3 / 2}} \mathrm{e}^{i(\boldsymbol{k} \cdot \boldsymbol{r})} .
$$

The spectral representation of this operator becomes

$$
\hat{T}^{(0)}\left(\boldsymbol{r}, \boldsymbol{r}^{\prime}\right)=\int \mathrm{d} \boldsymbol{k} \psi_{\boldsymbol{k}}(\boldsymbol{r}) T_{k}^{(0)} \psi_{\boldsymbol{k}}^{+}\left(\boldsymbol{r}^{\prime}\right),
$$

where the kernel of this operator

$$
T_{k}^{(0)}=\frac{(\hbar c)^{2}}{2 \mu c^{2}} k^{2} .
$$

Due to equivalence of eigenfunctions, the spectral representation of the relativistic kinetic energy operator is

$$
\hat{T}\left(\boldsymbol{r}, \boldsymbol{r}^{\prime}\right)=\int \mathrm{d} \boldsymbol{k} \psi_{\boldsymbol{k}}(\boldsymbol{r}) T_{k} \psi_{\boldsymbol{k}}^{+}\left(\boldsymbol{r}^{\prime}\right) .
$$

The kernel of this operator is defined by Equation (18):

$$
T_{k}=\mu c^{2}\left[\left(1-\left(\frac{\hbar c}{\mu c^{2}}\right)^{2} k^{2}\right)^{-1 / 2}-1\right] \text {. }
$$


The expectation value of the nonrelativistic kinetic energy in a given state of the internal two-particle Hamiltonian, whose eigenfunction is $\Psi(\boldsymbol{r})$, equals

$$
T^{(0)}=\int \mathrm{d} \boldsymbol{r} \int \mathrm{d} \boldsymbol{r}^{\prime} \Psi^{+}(\boldsymbol{r}) \hat{T}^{(0)}\left(\boldsymbol{r}, \boldsymbol{r}^{\prime}\right) \Psi\left(\boldsymbol{r}^{\prime}\right),
$$

while the expectation value of the relativistic kinetic energy is

$$
T^{(3)}=\int \mathrm{d} \boldsymbol{r} \int \mathrm{d} \boldsymbol{r}^{\prime} \Psi^{+}(\boldsymbol{r}) \hat{T}\left(\boldsymbol{r}, \boldsymbol{r}^{\prime}\right) \Psi\left(\boldsymbol{r}^{\prime}\right) .
$$

From operator definitions given by Equations ((29), (31) and (28)), alternative definitions of expectation values follow:

$$
T^{(0)}=\int \mathrm{d} \boldsymbol{k} \Psi^{+}(\boldsymbol{k}) T_{k}^{(0)} \Psi(\boldsymbol{k})
$$

and

$$
T^{(3)}=\int \mathrm{d} \boldsymbol{k} \Psi^{+}(\boldsymbol{k}) T_{k} \Psi(\boldsymbol{k}),
$$

where $\Psi(\boldsymbol{k})$ is Fourier image of wave function $\Psi(\boldsymbol{r})$ :

$$
\Psi(\boldsymbol{k})=\int \mathrm{d} \boldsymbol{r} \Psi(\boldsymbol{r}) \psi_{\boldsymbol{k}}^{+}(\boldsymbol{r}) .
$$

\section{The Harmonic Oscillator Potential}

One of the best known problems of nonrelativistic quantum mechanics, having successful applications, is a system of two particles with a harmonic oscillator potential. The internal Schrödinger equation for this system is

$$
\left(-\frac{(\hbar c)^{2}}{2 \mu c^{2}} \Delta+\frac{1}{2} \mu \omega^{2} r^{2}\right) \Psi=E^{(0)} \Psi,
$$

where $\mu$ is the reduced mass of the interacting particles. The equation has square integrable solutions

$$
\Psi_{n l m}(\boldsymbol{r})=N_{n l} x^{l} \exp \left(-x^{2} / 2\right) L_{n}^{l+1 / 2}\left(x^{2}\right) Y_{l m}(\vartheta \varphi)
$$

only for eigenvalues $E_{n l}^{(0)}=\hbar \omega(2 n+l+3 / 2)$. Here the argument of the radial function is $x=r / b$, with $b=\sqrt{\hbar /(\mu \omega)} . n=0,1,2, \cdots$ equals the number of nodes of the radial function and the normalization constant is

$$
N_{n l}^{2}=\frac{2 n !}{b^{3} \Gamma(n+l+3 / 2)} \text {. }
$$

To evaluate the expectation value of the corresponding relativistic kinetic energy operator $\langle\hat{T}\rangle_{n l}$, we require the expectation value of the nonrelativistic kinetic energy. According to Virial theorem for a harmonic oscillator potential

$$
\left\langle\hat{T}^{(0)}\right\rangle_{n l}=E_{n l}^{(0)} / 2=\langle\hat{V}\rangle_{n l},
$$

hence

$$
\frac{E_{n l}^{(0)}}{\mu c^{2}}=\beta(2 n+l+3 / 2) .
$$

Here and in the following expressions, for the sake of simplicity and comparison with different approximations, we use a dimension-free parameter 


$$
\beta=\frac{\hbar \omega}{\mu c^{2}} \text {. }
$$

The first order correction of the nonrelativistic value, defined in Equation (22), simplifies due to Virial theorem:

$$
\left\langle\left(\hat{T}^{(0)}\right)^{2}\right\rangle_{n l}=\left\langle\hat{V}^{2}\right\rangle_{n l}=\left(\frac{\hbar \omega}{2}\right)^{2}\left\langle x^{4}\right\rangle_{n l} .
$$

Thus, the value with the first order correction taken into account equals

$$
\begin{aligned}
\frac{E_{n l}^{(1)}}{\mu c^{2}}= & \beta(2 n+l+3 / 2) \\
& +\frac{3}{8} \beta^{2}\left[(2 n+l+3 / 2)^{2}+(2 n+l+3 / 2)+2 n(n+l+1 / 2)\right] .
\end{aligned}
$$

The second approximation, defined by Equation (23), gives:

$$
\frac{E_{n l}^{(2)}}{\mu c^{2}}=[1-\beta(2 n+l+3 / 2)]^{-1 / 2}-1+\beta(2 n+l+3 / 2) / 2 \text {. }
$$

In a nonrelativistic approximation $(\beta \ll 1)$, this expression leads to $E_{n l}^{(2)}=E_{n l}^{(0)}$. The new result of relativistic kinematics is the existence of a restricted set of eigenvalues, satisfying the condition

$$
2 n+l+3 / 2<\frac{1}{\beta} \text { and } \beta<2 / 3 .
$$

Finally, for the expectation value of the third approximation we need the Fourier image of the eigenfunction, defined by Equation (39). This problem is not complicated, since the transformation operator between the Hamiltonians of the harmonic oscillator in coordinates and momenta representations is well-known. These Hamiltonians, written in dimensionless variables, are correspondingly

$$
\begin{aligned}
& \hat{H}(\boldsymbol{r})=\frac{\hbar \omega}{2}\left(-\Delta_{\boldsymbol{x}}+\boldsymbol{x}^{2}\right), \\
& \hat{H}(\boldsymbol{p})=\frac{\hbar \omega}{2}\left(-\Delta_{\boldsymbol{y}}+\boldsymbol{y}^{2}\right) .
\end{aligned}
$$

Here $\boldsymbol{x}=\boldsymbol{r} / b$ and $\boldsymbol{y}=\boldsymbol{p} b / \hbar$. The mentioned above transformation operator is

$$
\frac{1}{(2 \pi)^{3 / 2}} \mathrm{e}^{i(x \cdot y)}
$$

Therefore, the eigenfunctions of these operators are connected by this transformation. The Fourier image of the original function is

$$
\Psi_{n l m}(\boldsymbol{p})=\bar{N}_{n l} y^{l} \exp \left(-y^{2} / 2\right) L_{n}^{l+1 / 2}\left(y^{2}\right) Y_{l m}(\bar{\vartheta} \bar{\varphi})
$$

where $\boldsymbol{y}=\boldsymbol{p} / d, \quad d=\hbar / b=\sqrt{\hbar \omega \mu}$ and $\boldsymbol{y}=(y, \bar{\vartheta}, \bar{\varphi})$. The normalization constant equals

$$
\bar{N}_{n l}^{2}=\frac{2 n !}{d^{3} \Gamma(n+l+3 / 2)} .
$$

This wave function with the kernel, defined in Equation (30) produces the 
expectation value of the nonrelativistic kinetic energy, equal $E_{n l}^{(0)} / 2$. According to the definition we express in Equation (36), the third approximation for relativistic kinetic energy equals

$$
\frac{T_{n l}^{(3)}}{\mu c^{2}}=\frac{n !}{\Gamma(n+l+3 / 2)} \int_{0}^{1 / \beta} \mathrm{d} z \cdot z^{l+1 / 2}\left[(1-\beta z)^{-1 / 2}-1\right] \mathrm{e}^{-z}\left[L_{n}^{l+1 / 2}(z)\right]^{2},
$$

and thus the approximation for total energy is

$$
\frac{E_{n l}^{(3)}}{\mu c^{2}}=\frac{T_{n l}^{(3)}}{\mu c^{2}}+\beta(2 n+l+3 / 2) / 2 .
$$

\section{The Coulomb Potential}

The system of a charged particle moving in a Coulomb field has many interesting applications. We consider a particle with charge $-e$ (electron or muon) moving in a Coulomb well, created by an atomic nucleus, whose mass equals $M$ and charge is $Z e$. The nonrelativistic Schrödinger equation for this system is

$$
\left(\frac{(\hbar c)^{2}}{2 \mu c^{2}} \Delta+\frac{\alpha \mathrm{Z} \hbar c}{r}+E_{n}^{(0)}\right) \psi_{n l m}(r \theta \varphi)=0 .
$$

Here $\mu$ is the reduced mass of light particle and atomic nucleus, $\alpha$ is the fine structure constant. The square integrable solutions of this equation:

$$
\psi_{n l m}(r \theta \varphi)=N_{n l} x^{l+1} \exp (-x / 2) L_{n-l-1}^{2 l+1}(x) Y_{l m}(\vartheta \varphi),
$$

where $x=2 r / n a, n=l+1, l+2, \cdots, \quad a=\hbar c / \alpha Z \mu c^{2}$,

$$
N_{n l}^{2}=\frac{(n-l-1) !}{a n^{2}(n+l) !}
$$

exists only for eigenvalues

$$
E_{n}^{(0)}=-\frac{\mu c^{2}}{2}\left(\frac{\alpha Z}{n}\right)^{2}
$$

Virial theorem for Coulomb potential gives

$$
\langle V\rangle_{n l}=2 E_{n},\left\langle\hat{T}^{(0)}\right\rangle_{n l}=-E_{n} .
$$

The first order correction, defined by Equation (22), is

$$
\frac{E_{n l}^{(1)}}{\mu c^{2}}=-\frac{1}{2}\left(\frac{\alpha Z}{n}\right)^{2}+\frac{3}{2}\left(\frac{\alpha Z}{n}\right)^{4}\left(\frac{n}{l+1 / 2}-\frac{3}{4}\right) .
$$

The correction, defined by Equation (23), equals

$$
\frac{E_{n l}^{(2)}}{\mu c^{2}}=\left[1-\left(\frac{\alpha Z}{n}\right)^{2}\right]^{-1 / 2}-\left(\frac{\alpha Z}{n}\right)^{2}-1 .
$$

Following from this equation, the condition of bound states existence in a Coulomb well looks like

$$
n>\alpha Z \text {. }
$$


For existing stable atomic nuclei $\alpha Z<1$, so relativistic kinematics do not restrict the number of bound states in a Coulomb well. On the other hand, significant relativistic corrections can arise for Hydrogen-like ions of heavy atoms.

Finally, for the evaluation of the correction, defined by Equation (36) we require the Fourier image of wave function $\psi_{n l m}(\boldsymbol{r})$. Corresponding expressions are complex enough, so we will only present results, obtained for the ground state function

$$
\psi_{100}(\boldsymbol{r})=\frac{\mathrm{e}^{-r / a}}{\sqrt{\pi a^{3}}} .
$$

The Fourier image of this function is

$$
\psi_{100}(\boldsymbol{k})=\frac{(2 a)^{3 / 2}}{\pi\left[1+(a k)^{2}\right]^{2}} .
$$

This function with kernel (30) gives a nonrelativistic kinetic energy, equal $-E_{10}^{(0)}=\mu c^{2}(\alpha Z)^{2} / 2$. With the relativistic kernel (32), we find the corrected energy value to be

$$
\frac{E_{10}^{(3)}}{\mu c^{2}}=\frac{32}{\pi} \int_{0}^{\frac{1}{\alpha Z}} \frac{x^{2}}{\left(1+x^{2}\right)^{4}}\left[\left(1-(\alpha Z x)^{2}\right)^{-1 / 2}-1\right]-(\alpha Z)^{2} .
$$

\section{Results}

The introduced method of relativistic corrections of bound state energies of twoparticle systems with harmonic oscillator potential presents the eigenvalues as functions of one dimensionless parameter $\beta<2 / 3$. Table 1 gives the different values of ground state energy, divided by $\mu c^{2}$, as functions of this parameternonrelativistic, defined by Equation (42), and three different approximations for relativistic dynamics, defined in Equations ((44), (45) and (53)). In the last column of Table 1 we have the energy obtained by the traditional method, when the nonrelativistic quantum mechanical momentum operator is defined as

Table 1. Different approximations of ground state energies in harmonic oscillator well.

\begin{tabular}{cccccc}
\hline$\beta$ & $\frac{E_{00}^{(0)}(\beta)}{\mu c^{2}}$ & $\frac{E_{00}^{(1)}(\beta)}{\mu c^{2}}$ & $\frac{E_{00}^{(2)}(\beta)}{\mu c^{2}}$ & $\frac{E_{00}^{(3)}(\beta)}{\mu c^{2}}$ & $\frac{E_{00}^{(-)}(\beta)}{\mu c^{2}}$ \\
\hline 0.001 & 0.0015 & 0.00150 & 0.00150 & 0.00150 & 0.00150 \\
0.01 & 0.0150 & 0.01514 & 0.01509 & 0.01515 & 0.01495 \\
0.1 & 0.1500 & 0.16406 & 0.15965 & 0.17199 & 0.14531 \\
0.2 & 0.3000 & 0.35625 & 0.34523 & 0.42617 & 0.28125 \\
0.3 & 0.4500 & 0.57656 & 0.57340 & 0.66631 & 0.40781 \\
0.4 & 0.6000 & 0.82500 & 0.88114 & 0.82711 & 0.52500 \\
0.5 & 0.7500 & 1.10156 & 1.37500 & 0.92913 & 0.63281 \\
0.6 & 0.9000 & 1.40625 & 2.61228 & 0.99864 & 0.73125 \\
0.666 & 0.9990 & 1.62275 & 31.1223 & 1.03511 & 0.79108 \\
\hline
\end{tabular}


corresponding to relativistic momentum, i.e. when the kinetic energy expansion

$$
T=\sqrt{m^{2} c^{4}+p^{2} c^{2}}-m c^{2}=\frac{p^{2}}{2 m}-\frac{p^{4}}{8 m^{3} c^{2}}+\cdots
$$

is approximated as

$$
\bar{T}=\sqrt{m^{2} c^{4}+p_{0}^{2} c^{2}}-m c^{2}=\frac{p_{0}^{2}}{2 m}-\frac{p_{0}^{4}}{8 m^{3} c^{2}}+\cdots
$$

From this energy expression, found in numerous textbooks and basic for Klein-Gordon and Dirac equations, it follows that

$$
E^{2}=\gamma^{2} m^{2} c^{4}=m^{2} c^{4}+p_{0}^{2} c^{2}=\left(1+\left(\frac{p_{0}}{m c}\right)^{2}\right) m^{2} c^{4},
$$

therefore it is not the completely relativistic expression for energy, but rather a crude approximation for the Lorentz factor

$$
\gamma=\frac{1}{\sqrt{1-v^{2} / c^{2}}}
$$

by $\sqrt{1+v^{2} / c^{2}}$. Therefore, the accuracy of this presentation does not exceed $\left(v^{2} / c^{2}\right)^{2}$. Moreover, this approximation results in the absence of relativistic momentum and energy dependence of $v$ in the limit $v \rightarrow c$. The corresponding expectation value for harmonic oscillator potential, given in the last column of Table 1, is

$$
\begin{aligned}
\frac{E_{n l}^{(-)}}{\mu c^{2}} & =\beta(2 n+l+3 / 2) \\
& -\frac{1}{8} \beta^{2}\left[(2 n+l+3 / 2)^{2}+(2 n+l+3 / 2)+2 n(n+l+1 / 2)\right] .
\end{aligned}
$$

The problem is to understand how sensitive to relativistic corrections are the energies of different quantum systems, performing vibrational movement. The first one among them is nuclear motion in diatomic molecules. The parameter $\beta$ takes a larger value at a small reduced mass of nuclei, hence the best system for the evaluation is the Hydrogen molecule $\mathrm{H}_{2}$. Corresponding reduced mass equals half the proton mass. The molecular internal energy is approximately the sum of the electromagnetic energy, the vibrational energy $(v+1 / 2) \hbar \omega$ and the rotational energy [7]. The characteristic value of vibration energy $\hbar \omega$ for this molecule equals approximately $0.5 \mathrm{eV}$. The parameter $\beta$ appears as very small, of the order $10^{-9}$, hence the relativistic corrections for the molecular vibration energy are negligible and can hardly be separated from other effects in observations. Next is the giant dipole resonance of nuclei; this resonance can be explained as the oscillations of protons and neutrons against each other with an energy of excitation given approximately by $E_{1}^{*}=77 A^{-1 / 3} \mathrm{MeV}$ [8]. Here $A$ is the mass number of the nucleus. The reduced mass of this mode equals approximately $470 \cdot A \mathrm{MeV}$, hence the parameter $\beta \approx 0.16 A^{-4 / 3}$. For nuclei with mass number $A \approx 60$ the parameter $\beta$ is of order $10^{-3}$.

For the ground state $(n=1, l=0)$ of Hydrogen-like ions, corresponding results are present in Table 2. For comparison with experimental data, the 
Table 2. Ionization potentials of Hydrogen-like ions $(\mathrm{eV})$.

\begin{tabular}{ccccccc}
\hline$Z$ & $J_{10}^{(0)}$ & $J_{10}^{(1)}$ & $J_{10}^{(2)}$ & $J_{10}^{(3)}$ & $J_{10}^{(-)}$ & Exp \\
\hline 1 & 13.6057 & 13.6030 & 13.6051 & 13.6030 & 13.6066 & 13.5984 \\
2 & 54.4228 & 54.3793 & 54.4141 & 54.3787 & 54.4373 & 54.4178 \\
3 & 122.4512 & 122.2313 & 122.4072 & 122.2266 & 122.5246 & 122.4544 \\
4 & 217.6911 & 216.9955 & 217.5519 & 216.9773 & 217.9229 & 217.7186 \\
5 & 340.1423 & 338.4442 & 339.8023 & 338.3912 & 340.7084 & 340.2260 \\
6 & 489.8049 & 486.2838 & 489.0996 & 486.1588 & 490.9787 & 489.9932 \\
40 & $21,769.11$ & $14,813.70$ & $20,271.28$ & $15,924.90$ & $24,087.58$ & $22,236.68$ \\
60 & $48,980.49$ & $13,768.75$ & $40,584.86$ & $29,189.06$ & $60,717.74$ & $51,515.58$ \\
70 & $66,667.89$ & $1,433.78$ & $49,936.25$ & $38,807.72$ & $88,412.60$ & $71,574.80$ \\
\hline
\end{tabular}

ionization potentials are expressed in electronvolts, equal to the energies with opposite sign. Experimental data, taken from [9], is given in the last column. Like in Table 1, the second column gives the nonrelativistic result (57), third, fourth and fifth-the approximations, defined by (59), (60) and (64) correspondingly. In the column, marked $J_{10}^{(-)}$the traditional approach is presented, defined by Equation (66) with first order correction of kinetic energy with opposite sign.

Table 2 shows that the nonrelativistic result $\left(J_{10}^{(0)}\right)$ is not far of the experimental value but requires slight corrections in all cases. The relativistic dynamics introduction cannot solve this problem, but is very useful as the first step. From our results it is apparent that both first order corrections-one with precise relativistic kinetic energy $\left(J_{10}^{(1)}\right)$ and second-traditional-with corrected kinetic energy expectation value smaller than nonrelativistic one $\left(J_{10}^{(-)}\right)$-give significantly different and in both cases not acceptable results. The two original methods of these corrections introduced above-the first one applying Lorentz multiplier $\left(J_{10}^{(2)}\right)$ and second applying spectral representation of relativistic kinetic energy operator $\left(J_{10}^{(3)}\right)$ demonstrate the necessity of further investigation of this problem, taking into account, among other things, the structure of atomic nucleus with defined charge and magnetism distributions.

An interesting conclusion follows from these results about the Hydrogen atom ionization potential. As usual, the starting point is the nonrelativistic value $J_{10}^{(0)}=13.6057 \mathrm{eV}$ with the traditional first order correction, predicting relativistic kinetic energy smaller than the nonrelativistic one, i.e.

$J_{10}^{(-)}=13.6066 \mathrm{eV}$. Comparing with the experimental data, this result is worse than zero approximation. The possibility of correction in approximation, proportional $(\alpha Z)^{4}$ is found-it is the so called Darvin term [10], considering the proton as a point-like particle. However, the structure of proton plays a significant role, as is shown by the proton charge radius measurement in muonic Hydrogen [11], giving a significantly different radius in comparison with proton radius, predicted by the traditional Hydrogen investigation [12]. Therefore, our approach, based on a precise relativistic kinetic energy definition, is more 
promising for further investigation of this problem.

\section{Conclusions}

Our investigation has shown that successful relativistic dynamics application needs Galilei invariant formalism for the description of a quantum system. Galilei invariance, basic for relativity, appears as a bridge between relativity and quantum mechanics for relativistic quantum systems theory. Internal Hamiltonian of the quantum system corresponds to the nonrelativistic dynamics of particles, performing mutual movement, when all particles are present in the same inertial system. No time coordinate and Lorentz transformations, describing relations between movements in different inertial systems, are necessary for these particles. The only modification of such a Hamiltonian, taking into account relativistic dynamics, is the introduction of the precise relativistic kinetic energy operator for every internal spatial variable instead of the corresponding nonrelativistic one. These operators, defined in [1] and above, are invariant with respect to translations, hence the modified Hamiltonian is Galilei invariant. Moreover, it has all symmetries of the nonrelativistic Hamiltonian. Therefore, the eigenfunctions of the relativistic Hamiltonian have the same exact quantum numbers-like parity, angular, spin and total momenta and so on-as eigenfunctions of the nonrelativistic internal Hamiltonian. This is because the only restrictions for these symmetries are introduced by the complexity of the quantum system's potential energy operator, having the same form in both cases because the relativistic mechanism of the interaction of particles does not play a role due to stationarity of the quantum system.

The introduced internal relativistic Hamiltonian in limit of small (in comparison with $\mu c^{2} / 2$ ) kinetic energies of particles appears as the nonrelativistic internal Hamiltonian. Both Hamiltonians for a system of identical particles are symmetrical with respect to particles' spatial coordinates and intrinsic degrees of freedom permutations. However, the antisymmetrization of the eigenfunctions of these Hamiltonians is problematic, because permutations of particles coordinates produce linear transformations of Jacobi variables. Traditional methods of this operation are not applicable. It is necessary to apply original methods, whose historical overview and the simplest known solution of the problem is present in [6].

Our investigation has shown that known potentials of particles interactions are weak enough to produce the quantum system with significant relativity. As a result, the relativity is soft, so it is acceptable to apply the introduced approximate methods for correction of nonrelativistic energies of quantum systems bound states.

The really interesting quantum system, whose solution needs more sophisticated relativistic effects consideration, is the nucleon. For weakly bound states (such as proton and neutron) to exist in a deep confining well, significant kinetic energy is necessary, a few times larger than the relativistic limit. Therefore, for the solution of this problem, application of relativistic theory is 
very promising.

\section{References}

[1] Kamuntavičius, G.P. and Kamuntavičius, G. (2017) Kinematic Relativity of Quantum Mechanics: Free Particle with Different Boundary Conditions. Journal of Ap plied Mathematics and Physics, 5, 853-861. https://doi.org/10.4236/jamp.2017.54075

[2] Das, A. (1993) The Special Theory of Relativity. Springer, New York. https://doi.org/10.1007/978-1-4612-0893-8

[3] Messiah, A. (1965) Quantum Mechanics. Vol. 2, North-Holland, Amsterdam.

[4] Bohr, A. and Mottelson, B.R. (1969) Nuclear Structure. Vol. 1, Benjamin Inc., New York.

[5] Fodor, Z. and Hoelbling, C. (2012) Light Hadron Masses from Lattice QCD. Reviews of Modern Physics, 84, 449-495. https://doi.org/10.1103/RevModPhys.84.449

[6] Kamuntavičius, G.P. (2014) Galilei Invariant Technique for Quantum System Description. Journal of Mathematical Physics, 55, Article ID: 042103. https://doi.org/10.1063/1.4870617

[7] Levine, I.N. (2009) Quantum Chemistry. 6th Edition, Pearson Prentice Hall, London.

[8] Henley, E.M. and Garcia, A. (2007) Subatomic Physics. 3rd Edition, World Scientific, New Jersey. https://doi.org/10.1142/6263

[9] Kramida, A., Ralchenko, Y., Reader, J. and NIST ASD Team (2014). NIST Atomic Spectra Database (ver.5.2). Available: http://physics.nist.gov/asd

[10] Manoukian, E.B. (2006) Quantum Theory. Springer, Dordrecht.

[11] Pohl, R., et al. (2013) The Size of the Proton. Nature (London), 466, 213-216. https://doi.org/10.1038/nature09250

[12] Patrignoni, C., et al. (Particle Data Group) (2016) Review of Particle Physics. Chinese Physics C, 40, Article ID: 100001.

\section{Submit or recommend next manuscript to SCIRP and we will provide best} service for you:

Accepting pre-submission inquiries through Email, Facebook, LinkedIn, Twitter, etc. A wide selection of journals (inclusive of 9 subjects, more than 200 journals)

Providing 24-hour high-quality service

User-friendly online submission system

Fair and swift peer-review system

Efficient typesetting and proofreading procedure

Display of the result of downloads and visits, as well as the number of cited articles

Maximum dissemination of your research work

Submit your manuscript at: http://papersubmission.scirp.org/

Or contact jamp@scirp.org 